\title{
Pediatric Nephrology: Update for Clinicians
}

\author{
Aditi Sinha $^{1} \cdot$ Arvind Bagga $^{1}$
}

Received: 14 April 2020 / Accepted: 14 April 2020 / Published online: 28 May 2020

(C) Dr. K C Chaudhuri Foundation 2020

The last symposium on pediatric nephrology was published more than a decade ago in the Journal. The last decade has seen a paradigm shift in diagnosis and management of kidney diseases. We now have consensus on definitions and severity of acute kidney injury and chronic kidney disease. This has enabled collection of data and implementation of studies with uniform inclusion criteria, and emergence of evidence-base for therapy. Collaborative efforts have resulted in more precise histological definitions of lupus nephritis, IgA nephropathy, C3 glomerulopathy and transplant rejection. Remarkable advances have been made in the pathogenesis of cystic kidney diseases, renal tubular disorders, nephrotic syndrome and hemolytic uremic syndrome. An important advance has also been the understanding of an inextricable link of genetic mutations as the basis for multiple diseases of the kidneys and urinary tract. Finally, the last decade has seen the emergence and application of newer immunosuppressive agents, including monoclonal antibodies, for a spectrum of glomerular disorders, ranging from nephrotic syndrome and glomerulonephritis, to vasculitis and hemolytic uremic syndrome.

The Symposium will be published in two issues. The first issue carries topics on common disorders in clinical nephrology. Acute kidney injury (AKI) is common in critically ill children and affects $\sim 30-40 \%$ patients admitted to pediatric intensive care units. We are honored to have Dr. Prasad Devarajan, a highly respected pediatric nephrologist with exceptional work in the specialty, review advances in diagnosis, risk stratification, prevention and management of AKI [1]. The next article by Rupesh Raina and colleagues discusses renal replacement strategies for children with AKI. They highlight the indications, modes of access and application of peritoneal dialysis, hemodialysis, continuous renal replacement

Arvind Bagga

arvindbagga@hotmail.com

Aditi Sinha

aditisinhaaiims@gmail.com

1 Department of Pediatrics, All India Institute of Medical Sciences, New Delhi 110029, India therapy and sustained low-efficiency dialysis for children with AKI [2]. The evaluation of etiology of hematuria is a challenge. A cause is often found in patients with symptomatic gross hematuria, especially in context of acute glomerulonephritis or renal stones. Evaluation of patients with persistent asymptomatic hematuria, with or without proteinuria, often requires detailed and expensive studies. Aparna Iyengar and her colleague emphasize a practical and algorithm based approach towards making an appropriate diagnosis [3].

Urinary tract infections (UTI) are an important bacterial infection of childhood. Diagnosis and long-term management of UTI continues to be a controversial topic in nephrology. Ranjeet Thergaonkar and Pankaj Hari review the current diagnosis, therapy and subsequent evaluation of UTI [4]. They also discuss the features, diagnosis and management of bowel bladder dysfunction and vesicoureteric reflux, both being risk factors for recurrent UTI and reflux nephropathy. The last article in the first part of the symposium focuses on the pathophysiology and management of edema, a commonly encountered problem in children (and adults) with difficult-to-treat nephrotic syndrome. The authors describe current perspectives in management of moderate to severe edema, and propose a management strategy for these patients [5].

The second part of the Symposium provides an overview of the role of genetics in evaluation of kidney diseases, and focuses on three important groups of renal tubular disorders. The editors of this symposium are grateful to Dr. Ishwar Verma and his team for their article on genetic testing in kidney diseases [6]. They emphasize the value of next-gen sequencing technology in the diagnosis of kidney diseases, especially steroid resistant nephrotic syndrome, congenital anomalies of the kidney, cystic kidney disease, tubulopathies, nephronophthisis, and atypical hemolytic uremic syndrome. The high rate of identifiable genetic causes, and the fact that some of them might target very specific aspects of tubular (or glomerular) function, makes them targets for gene-editing in the future.

The next article by Amita Sharma and colleagues discusses the epidemiology, clinical features, and work-up required for nephrolithiasis [7]. They highlight the importance of a thorough metabolic evaluation, stone analysis and exome 
sequencing in ascertaining the cause of kidney stones, and the strategies and choice of surgical interventions. In the following article, Sushil Kabra and colleagues discuss the differential diagnosis of hypokalemic metabolic alkalosis. The authors emphasize the genetic abnormalities that result in Bartter and Gitelman syndromes, and underscore the importance of cystic fibrosis and congenital chloride diarhhea in mimicking these disorders [8]. The last article updates the review on renal tubular acidosis (RTA), authored by the editors, in 2007 . We discuss current views on pathophysiology, including understanding of the genetic basis of primary and secondary forms of RTA, and an approach to evaluation and management [9].

We are grateful to the authors, each of whom is an icon in their respective fields, for their scholarly contributions to this Symposium. The articles are of high quality and provide stateof-art reviews on respective topics. We are sure that the readers will find the articles useful, and hopefully make up for the long gap since the last symposium!

\section{Compliance with Ethical Standards}

Conflict of Interest None.

\section{References}

1. Roy J-P, Devarajan P. Acute kidney injury: diagnosis and management. Indian J Pediatr. 2019. https://doi.org/10.1007/s12098-01903096-y.
2. Sethi SK, Chakraborty R, Joshi H, Raina R. Renal replacement therapy in pediatric acute kidney injury. Indian J Pediatr. 2020. https:// doi.org/10.1007/s12098-019-03150-9.

3. Vedula R, Iyengar AA. Approach to diagnosis and management of hematuria. Indian J Pediatr. 2020. https://doi.org/10.1007/s12098020-03184-4.

4. Thergaonkar RW, Hari P. Current management of urinary tract infection and vesicoureteral reflux. Indian J Pediatr. 2019. https://doi. org/10.1007/s12098-019-03099-9.

5. Meena J, Bagga A. Current perspectives in management of edema in nephrotic syndrome. Indian J Pediatr. 2020. https://doi.org/10.1007/ s12098-020-03252-9.

6. Arora V, Anand K, Verma IC. Genetic testing in pediatric kidney disease. Indian J Pediatr. 2020. https://doi.org/10.1007/s12098-02003198-y.

7. Ang AJS, Sharma AA, Sharma A. Nephrolithiasis: approach to diagnosis and management. Indian J Pediatr. 2020. In Press.

8. Mantoo MR, Kabra M, Kabra SK. Cystic fibrosis presenting as Pseudo-Bartter syndrome: an important diagnosis that is missed! Indian J Pediatr. 2020. https://doi.org/10.1007/s12098-020-03342-8.

9. Bagga A, Sinha A. Renal tubular acidosis. Indian J Pediatr. 2020. https://doi.org/10.1007/s12098-020-03318-8.

Publisher's Note Springer Nature remains neutral with regard to jurisdictional claims in published maps and institutional affiliations. 